\title{
A New Class of Solar Oscillation Measurements
}

\section{J. Schou}

\author{
Stanford University, HEPL Annex A201, Stanford, CA 94305-4085, \\ $U S A$
}

\begin{abstract}
Over the past few years the quality and quantity of basic helioseismic data have increased dramatically as instruments such as MDI on the SOHO spacecraft and the GONG network have become operational. The data from these new instruments have led to a significant increase in our ability to make inferences about the solar interior, and resulted in a long series of often surprising and spectacular discoveries. In this review I describe some of the most recent results and what progress we may be able to make in the near future with improved analysis methods and with a better understanding of solar oscillations and the instruments used to observe them.
\end{abstract}

\section{Introduction}

With the improvement in the quality of basic helioseismic data from the MDI and GONG projects, compared to that from earlier experiments, we have started to see finer details in the internal structure and rotation of the Sun and how some of these features change over time. In this paper I start by presenting some of these results and discuss how they may relate to each other.

However, we have also come to realize that with improved data one can detect systematic errors too small to see in the earlier datasets and that we thus need to improve our understanding of how the instruments work and how our analysis algorithms need to take this, as well as the subtle details of the solar oscillation spectra, into account.

Since the local helioseismic techniques and some of their results are reviewed elsewhere in these proceedings I will not present those results here. Also this paper is mostly a review of the results and the techniques used, rather than an attempt at making physical interpretations.

\section{Data Sets}

Most of the recent progress in detecting solar cycle changes has come from analysis of data from the GONG and MDI projects. To provide background I will start with a brief description of these datasets and some of the important differences between the instruments and analysis methods used. Some of the implications of these differences are described later. 


\subsection{MDI}

The Michelson Doppler Imager (MDI) on the SOHO spacecraft is primarily designed to observe the Sun in Doppler velocity at high spatial resolution, including full disk images with $1024^{2}$ resolution. As part of the MDI observing program low resolution (200x200 pixels) spatially averaged Dopplergrams are produced with a very high duty cycle $(\approx 99 \%)$. This so-called Medium- $l$ program was used for the MDI results presented here.

The MDI processing of these data produces non-overlapping 72 day timeseries for degrees $l$ up to 300. These time-series are analyzed by the algorithm described in Schou (1992) to extract the mode parameters. The MDI analysis method fits the Fourier transforms for all $m$ 's at each $n, l$ simultaneously using the results from other multiplets to estimate their leaks. This method uses leakage matrices calculated from our knowledge of the properties of the solar oscillations and the instrument. For the results presented here so-called $a$ coefficients were fitted directly rather than by estimating the frequencies first. The $a$ coefficients are the coefficients in an expansion of the mode frequencies $\nu_{n l m}$

$$
\nu_{n l m}=\nu_{n l}+\sum_{j=0}^{j=j_{\max }} a_{j}(n, l) P_{j}^{(l)}(m)
$$

where $\nu_{n l}$ is the mean mode frequency, $a_{j}(n, l)$ the $a$ coefficients and $P_{j}^{(l)}$ suitable polynomials of order $j$ (see Schou, Christensen-Dalsgaard, \& Thompson 1993).

\subsection{GONG}

The Global Oscillation Network Group (GONG) uses a collection of six sites around the Earth to collect moderate resolution $\left(\approx 256^{2}\right.$ pixels $)$ Doppler images with a high duty cycle $(\approx 90 \%)$. Due to the limited resolution of the instruments and the aliasing present, time-series are only produced up to $l=200$ and the standard analysis is only performed up to $l=150$. The GONG time-series are analyzed in 108 day pieces on 36 day centers by the method described in Hill et al. (1996). This method fits each $n, l, m$ and the leaks in each spectrum separately. The resulting frequencies are then weeded and $a$ coefficients are fitted to the remaining mode frequencies. This method does not rely on a leakage matrix and makes no use of the information from fits of modes other than the target. Furthermore, it implicitly assumes that each primary peak $(n, l)$ or leak contains only one $m$ value.

\section{Results}

The recent results from MDI and GONG have mostly been regarding the temporal evolution of the solar rotation and thermal structure. With roughly 5 years of data we finally have densely sampled datasets covering a significant fraction of a solar cycle and as the results presented in the following subsections demonstrate, we are starting to see intriguing variations in several areas. 

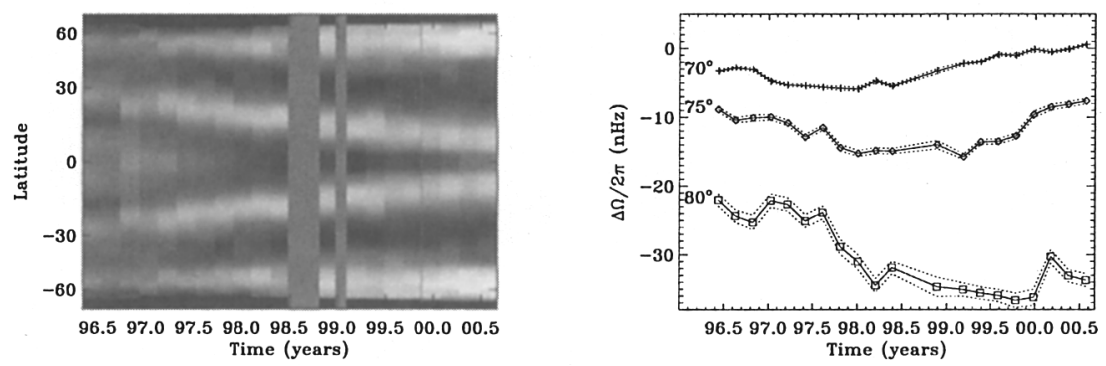

Figure 1. Left (a): The near surface rotation rate as deduced from MDI $f$ modes with a second order polynomial in $\sin ^{2} \theta$ subtracted. The gray scale is linear between $\pm 9 \mathrm{~m} / \mathrm{s}$. Right (b) rotation rate near at three near polar latitudes as a function of time. Error bars are $1 \sigma$.

\subsection{Rotation}

The two main areas where temporal changes in the rotation rate have been detected are near the surface and at the interface between the radiative interior and the convection zone (the tachocline). Since the tachocline variations are described elsewhere in these proceedings (Howe et al. 2000a) I will concentrate on the near surface variations in this review.

To illustrate some of these results, Figure 1a shows the near surface rotation rate with a second order polynomial in $\sin ^{2} \theta$ ( $\theta$ being the co-latitude) subtracted. These flows were determined, as described in Schou (1999), by, for each 72 day time interval, averaging the $a$ coefficients for all the available $f$ modes with $160 \leq l \leq 250$ and performing an OLA inversion for the rotation rate as a function of latitude. These results are basically an average of the rotation rate in the outer $1 \%$ of the Sun by radius.

The dominant feature seen is the bands of faster and slower rotation slowly migrating towards to equator. These so-called torsional oscillations have been observed previously using surface Doppler shift measurements (e.g. Ulrich 1998). The helioseismic measurements have allowed us to both confirm the earlier measurements and to probe their variations with depth (Howe et al. 2000b), as illustrated in Figure 2. As can be seen from this Figure the signal is visible to a significant depth. However, tests with artificial data (Howe et al. 2000b) show that the signal at the deepest radii shown if Figure 2 are due to the limited radial resolution and that the flows most likely don't extend below $0.92 R_{\odot}$.

Another feature of the near surface rotation rate is the rapid decrease near the poles visible in Figure 1a. As can be seen, this decrease is well in excess of the three term polynomial in $\sin ^{2} \theta$. This is further illustrated in Figure 1b which shows the rotation rate as a function of time at three latitudes. The perhaps most interesting feature seen near the poles in Figure 1a is that the border of the slow rotation appears to be moving towards the poles, especially towards the end of the time interval. This is consistent with Figure $1 \mathrm{~b}$ which shows that the rotation has a minimum, as a function of time, at all the latitudes shown, but that the time of the minimum is moving towards later times at higher latitudes. 

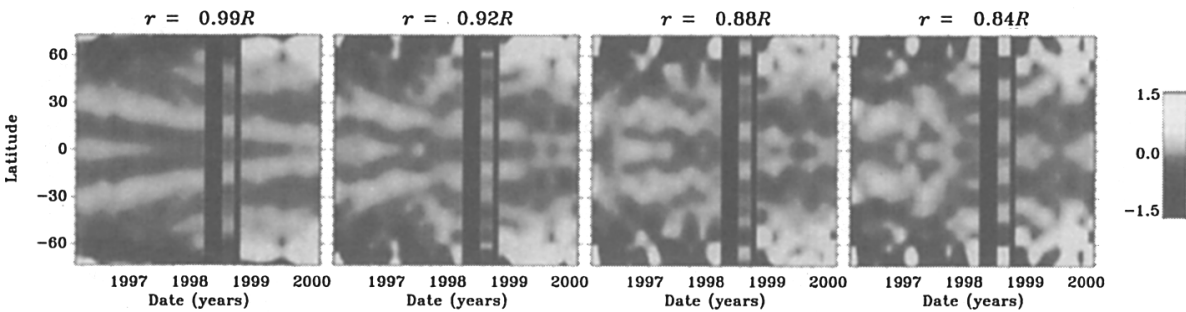

Figure 2. Inversions (from Howe et al. 2000b) showing the results from inverting MDI $\mathrm{p}$ and $\mathrm{f}$ mode data at selected depths. Grayscale is from $-1.5 \mathrm{nHz}$ to $+1.5 \mathrm{nHz}$.

\subsection{Sound Speed Variations}

The other main area where changes are seen is in the structure as a function of latitude. Since it is in practice impossible to distinguish between different sources of the asphericity using the measured frequencies, the perturbation is generally parameterized as a relative change in the effective sound speed $(\delta c / c)$.

Figure 3 shows averages of the even $a$ coefficients as a function of the corresponding expansion coefficients (with latitude) of the magnetic flux (see Antia et al. 2000a,b for details). As can be seen the even $a$ coefficients appear to be tracking the surface magnetic field almost perfectly. This is consistent with earlier results showing that the asphericity is largely a surface phenomenon.

To study the asphericity further, inversions have been performed to determine if temporal changes can be seen in the solar interior (Dziembowski et al. 2000; Antia et al. 2000a,b). While there are indications of below surface changes, the results have so far only been marginally significant. On the other hand the temporal average, shown in Figure 4, shows that the effective sound speed is not independent of latitude and that there is a localized increase in the sound speed around a radius of $0.90 R_{\odot}$ and a latitude of $60^{\circ}$.

Finally Figure 5 shows the surface term, subtracted as part of the inversion, as well as contours of the unsigned flux. The correspondence is very good, not entirely surprising given Figure 4. However, it is worth noting that there is a signal in the sound speed perturbation even when there is very little field. The near polar features appear to be moving towards the poles in a manner similar to that seen in the rotation rate (Fig. 1).

\section{Discussion and Conclusion}

As described above we have been able to detect temporal changes in both the solar rotation rate and structure and in some cases these changes have been resolved in depth. The changes described here have been fairly close to the surface, however, the perhaps most interesting and surprising result, discussed elsewhere in these proceedings (Howe et al. 2000a), is signs of changes in the rotation rate near the bottom of the convection zone with a period of 1.3 years. Despite several attempts (e.g. Monteiro 2000) at detecting changes in the structure near the bottom of the convection zone, the results have, so far been inconclusive. 

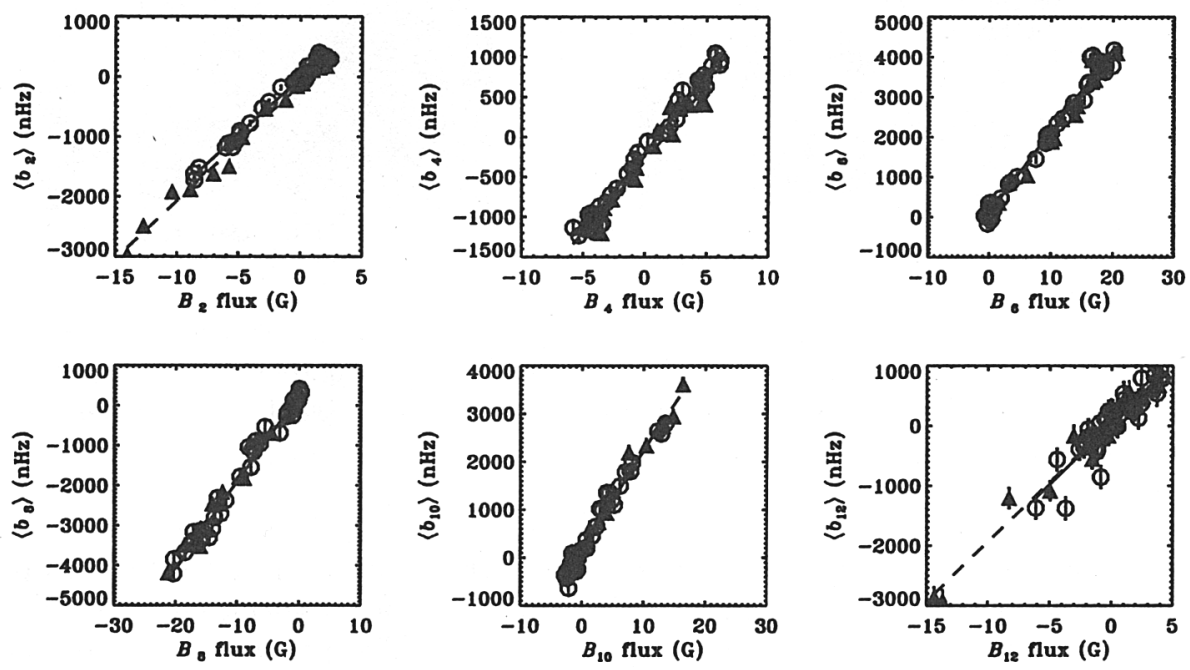

Figure 3. Weighted averages of the even $a$ coefficients as a function of the corresponding components of a Legendre expansion of the magnetic flux from Kitt Peak synoptic maps. Circles are for GONG and triangles for MDI. The respective fits are shown by solid and dashed lines. For details see Antia et al. (2000a,b).
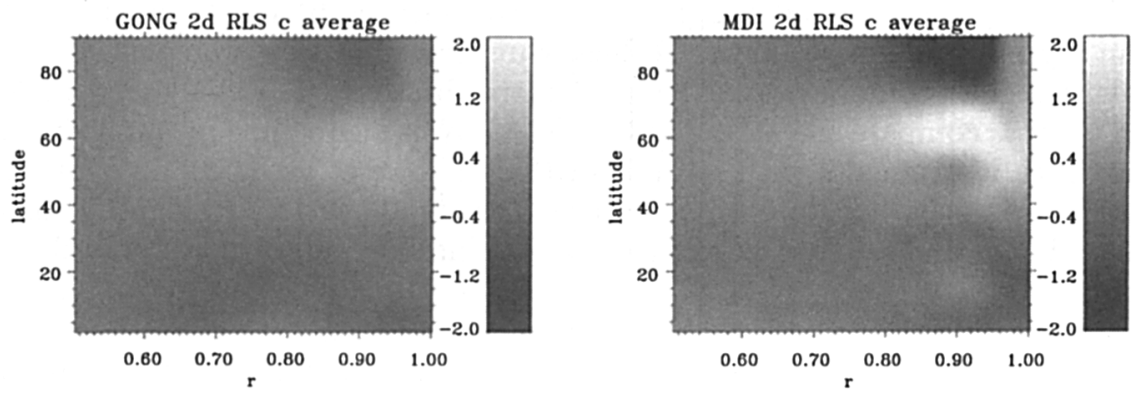

Figure 4. Average sound speed perturbation $(\delta c / c)$ as a function of fractional radius and latitude. Units are $10^{-4}$. For details see Antia et al. $(2000 a, b)$. 

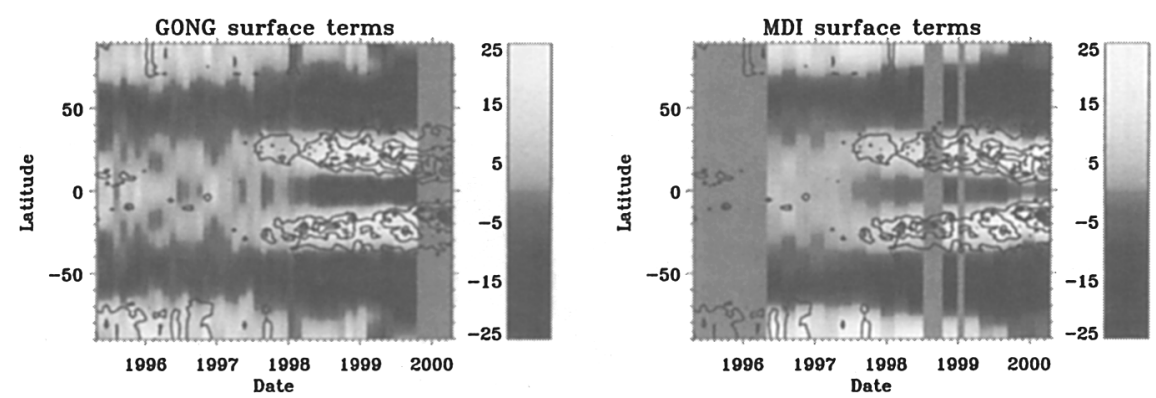

Figure 5. The surface term from the inversion shown in Fig. 4 as a function of time and latitude. Units are $10^{-3}$. Also shown as contours is the Kitt Peak unsigned flux with the mean subtracted. Contour spacing is $10 \mathrm{G}$.

An interesting common feature seen in both the near surface rotation rate and structure is the polar features. In both cases a perturbation is seen to propagate towards the pole (see Figures 1 and 5 . The physical origin of this perturbation has not been determined, but one might suspect that it is somehow related to the dynamo often presumed to involve the subduction of surface fields near the poles.

On the other hand it has also become clear that our ability to make inferences about the solar interior is in many cases limited by our analysis methods and in particular by our modeling of various instrumental imperfections. While the GONG and MDI results generally agree quite well, there are many subtle (and sometimes obvious) differences, as illustrated by Figure 4.

Many of these differences can be traced back to well understood and largely harmless problems, such as the different spatial resolution of the instruments and the resulting different coverage in the $l-\nu$ diagram. However, others are clearly due to incomplete modeling of the instruments (such as distortions) or problems with the analysis algorithms used (such as fitting symmetric rather than asymmetric line profiles). To better understand these problems efforts are underway both to understand the instruments better (e.g. Rabello-Soares 2000) and to cross compare the MDI and GONG analysis algorithms by analyzing data from both instruments with both algorithms (Howe et al. 2000c). While these studies are not yet completed, they have already allowed us to identify some of the problems and we thus expect to have significantly improved data in the near future.

Having resolved these problems we may also finally be able. to accurately estimate mode parameters at high frequencies and degrees which will allow us to determine the near surface rotation and structure accurately.

While these problems have prevented us from fully utilizing the data, the discoveries made so far have been spectacular and in many cases totally unexpected. Since we expect to understand most of these problems substantially better shortly we may thus expect more spectacular results in the near future.

An obvious future improvement is that as time goes by we will have better statistics. For the temporal mean this will mostly be a $\sqrt{n}$ effect, and thus 
rather slow. For the temporal variations, on the other hand, the improvement will be much faster, in particular since we have not yet observed for a full 11 year solar cycle.

The other major improvement will be data from new and improved instruments. The GONG network will shortly be upgraded to use a $1024^{2}$ detector which will allow it to not only cover all the global modes (i.e. those that have not blended into ridges) without aliasing, but also to observe modes up to $l=1000$ which will make it possible to perform ridge fits as well as local helioseismology.

In space the Solar Dynamics Observatory (SDO) is expected to be launched within the next decade. This spacecraft will, among other things, be able to observed the Sun continuously at very high spatial resolution $(4 \mathrm{Kx} 4 \mathrm{~K})$. Unlike MDI, SDO will have the ability to downlink all the images, rather than only selected observables for part of the time, allowing us to further improve our diagnostic capabilities.

While going to very high resolution will allow us to use high degree modes not previously accessible, and thereby allow us to study the surface layers in greater detail, these new instruments are not likely to provide a substantial improvement in our ability to measure low and medium degree modes, and thus their main function, as far as these modes are concerned, is to extend the available length of high quality observations. To increase the number of low and medium degree modes available to our analysis (such as finally detecting $\mathrm{g}$ modes) or to substantially increase the signal to noise ratio, is likely to require observing a larger fraction of the solar surface, to improve our current measurement techniques and/or to use entirely different methods for detecting the modes (such as space interferometers to detect the g-modes).

However, the most spectacular discoveries in helioseismology in the next few years are likely to be something unexpected or to be discovered using methods not even thought of at this time. With luck we may soon discover how the activity in the Sun operates. This will have profound impacts, not just on solar physics, but on our understanding of the space environment in which our Earth exists.

Acknowledgments. SOHO is a project of international cooperation between ESA and NASA. This work was funded by grant NAG5-8878 to the SOI/MDI project at Stanford University. The author wishes to thank the authors of the papers cited for sharing their figures, some before publication. In particular I wish to thank Rachel Howe for providing versions of the Figures suitable for inclusion into this paper.

\section{References}

Antia, H. M., et al. 2000, MNRAS, submitted

Antia, H. M., et al. 2000b, in Proc. Helio- and Asteroseismology at the Dawn of the Millennium, ed. A. Wilson, ESA SP-464 (Noordwijk: ESA), submitted

Dziembowski, W. A. 2000 ApJ, 537, 1026

Hill, F., et al. 1996, Science, 272, 1292

Howe, R., et al. 2000a, these proceedings 
Howe, R., et al. 2000b, ApJL, 533, 163

Howe, R., et al. 2000c, in Proc. Helio- and Asteroseismology at the Dawn of the Millennium, ed. A. Wilson, ESA SP-464 (Noordwijk: ESA), submitted

Monteiro, M. J. P. F. G. 2000, in Proc. Helio- and Asteroseismology at the Dawn of the Millennium, ed. A. Wilson, ESA SP-464 (Noordwijk: ESA), submitted

Rabello-Soares, M. C. 2000, in Proc. Helio- and Asteroseismology at the Dawn of the Millennium, ed. A. Wilson, ESA SP-464 (Noordwijk: ESA), submitted

Schou, J. 1992, PhD Dissertation, Aarhus University

Schou, J. 1999, ApJL, 523, 181

Schou, J., Christensen-Dalsgaard, J., \& Thompson, M. J. 1993, ApJ, 433, 389

Ulrich, R. K. 1998, in Proc. SOHO6/GONG98 Workshop, Structure and Dynamics of the Interior of the Sun and Sun-like Stars, ed. S. G. Korzennik \& A. Wilson, ESA SP-418 (Noordwijk: ESA), 851 